\title{
Hocus POCUS in Nephrology: is it time for magic?
}

\author{
Hugo Diniz ${ }^{1,2}$, Luís Coentrão ${ }^{1,2,3}$ \\ ${ }^{1}$ Nephrology Department, Centro Hospitalar e Universitário de São João, Oporto, Portugal \\ ${ }^{2}$ Nephrology \& Infectious Diseases R\&D, i3S - Instituto de Investigação e Inovação em Saúde da Universidade do Porto, Oporto, Portugal \\ ${ }^{3}$ Faculty of Medicine, University of Oporto, Oporto, Portugal
}

Point-of-care ultrasound (POCUS) is an emerging bedside discipline that aims to enhance physical examination findings with real-time information from the ultrasound. As opposed to a comprehensive examination of an anatomic area performed by a radiologist, POCUS is intended to answer focused clinical questions and is performed by the same physician examining the patient. Recently, POCUS has been described as the fifth pillar of physical examination in addition to inspection, palpation, percussion, and auscultation ${ }^{1}$. In the United States of America, POCUS training is well established in emergency medicine and intensive care. In Portugal, POCUS is taking its first steps, mainly in Internal Medicine, with the creation of the NEEco (Núcleo de Estudos de Ecografia) of the Portuguese Society of Internal Medicine. Interestingly, it is also being integrated into medical school training, as one of the objectives of the School of Medicine of Universidade do Minho is to put a pocket ultrasound in the hands of each of their students starting in the year 2020-21.

Ultrasound exposure in nephrology was mostly confined to procedural guidance, but POCUS is quickly expanding the scope of ultrasound in our specialty. For example, one can quickly exclude hydronephrosis using POCUS at the time of history taking, which reduces fragmentation of care and enhances the treatment of patients with acute kidney injury. Further, maturation of the arteriovenous fistula can be assessed during regular nephrology clinic visits, speeding up vascular access care. POCUS can also be used to identify peritoneal dialysis catheter tunnel-infection and monitor response to therapy.

Determination of volume status is a challenging area of nephrology practice, one in which we think POCUS can truly shine. Traditional physical examination findings are known to be poor discriminators of volume overload in our patients ${ }^{2}$. POCUS enhances our ability to detect congestion through the use of lung ultrasound, vascular ultrasound, focused echocardiography, and more advanced hemodynamic assessments such as portal vein pulsatility or renal venous impedance index. For example, lung ultrasound can detect B-lines, discrete laser-like vertical hyperechoic artifacts that arise from the pleural line, which provide a semi-quantitative estimation of extravascular lung water ${ }^{3}$. Focused echocardiography can provide information on left ventricular function, right ventricular size, or inferior vena cava collapsibility, which can be used to discriminate between a normal/low central venous pressure (CVP) and a high CVP 4 . Vascular ultrasound can be used for a better evaluation of the internal jugular vein, and quickly estimate the venous jugular pulse. As the evidence piles up that congestion is a key driver of adverse outcomes in the cardiorenal population, POCUS will have huge implications in this field, by providing an accessible, non-invasive tool for objective assessment of fluid excess and monitoring of response to an intervention such as ultrafiltration or diuretic therapy.
It is important to recognize that while POCUS can obviate the need for further investigation in some cases, it is not a replacement for formal ultrasonography. For example, while a nephrologist can perform focused echocardiography, it cannot be equated to a comprehensive echocardiogram performed by cardiology which is used to assess detailed wall motion and valvular function. Equally so, radiologists will continue to be the experts in the systematic ultrasonography of the kidneys and urinary tract. POCUS-performing nephrologists are expected to have some knowledge of the adjacent anatomy and discuss any abnormal or doubtful findings with other specialties so that appropriate follow-up studies can be undertaken.

Despite the existence of short-term POCUS training opportunities in Portugal and abroad, fellowship-based longitudinal training provide better results regarding long-term skill retention. Unfortunately, most general nephrology training programs have not yet fully embraced the rapidly evolving field of POCUS. John Hopkins University recently proposed a 2-week POCUS curriculum for nephrology fellows, which led to substantial improvement in trainees' skills in both subjective and objective assessments ${ }^{5}$. Based on their experience, the Nephrology Division of the University of Florida suggested that an 8-week period spread out over two years is needed to accommodate both the didactic training and scanning experience required to achieve competence ${ }^{6}$.

How can we start building a nephrology POCUS community in Portugal? In addition to attending workshops organized by professional societies, a multidisciplinary approach in collaboration with other specialties with bedside or POCUS experience may prove beneficial in the initial stages of designing a curriculum. Having dedicated POCUS lectures or workshops during Nephrology meetings such as Encontro Renal, like the American Society of Nephrology's Kidney Week would increase exposure and interest in this emerging field. The Portuguese Society of Nephrology could found a POCUS group to create a curriculum, coordinate training opportunities, and help with the certification and credentialing of the Portuguese divisions. Ensuring the quality of training is of the utmost importance because POCUS is both an operator-dependent technique and its findings have to be put into clinical context. In an ideal future, every nephrology fellowship program should have a faculty "champion" with ultrasound experience who leads the POCUS training and collaborates with other specialties.

Along with the lack of training programs, another limiting factor to the widespread adoption of POCUS is the availability of ultrasound equipment, as not every Portuguese nephrology department has access to portable or semi-portable ultrasound equipment. Sharing 
the costs with other interested divisions could be helpful. Moreover, highly portable hand-held ultrasound devices are getting more affordable every day, and offer most of the commonly used functionalities, such as color Doppler. This is probably the best option to start a POCUS program, as they are the cheapest alternative, can be used in every corner of the hospital and allow sharing of images with other colleagues or specialties.

In addition to enhancing our bedside examination and diagnostic skills, there is increasing evidence that the use of POCUS improves cardiorenal outcomes. Although the data is still scarce, in a randomized controlled trial the use of lung ultrasound was associated with a reduction in heart failure hospitalizations ${ }^{7}$. Another recent trial showed that lung ultrasound is an effective way to guide dry-weight reduction in hemodialysis patients and is associated with better blood pressure control ${ }^{8}$. Also, as we move towards a value-based healthcare reimbursement system, POCUS can result in more judicious utilization of the resources and enhanced patient satisfaction, which can result in savings to the healthcare system. Finally, the widespread adoption of POCUS in nephrology could also increase the attractiveness of our specialty, which lacks procedures compared to other internal medicine subspecialties such as cardiology and gastroenterology.

POCUS has the potential to impact our practice in such a big way that insonation might become part of our routine physical examination. Despite all the challenges ahead of us, we should seize the opportunity and become leaders in this emerging field. The time for nephrologists to yield the ultrasound "magic wand" has come.

Disclosure of potential conflicts of interest: none declared

\section{References}

1. Narula J, Chandrashekhar Y, Braunwald E. Time to add a fifth pillar to bedside physical examination: Inspection, palpation, percussion, auscultation, and insonation. JAMA Cardiol [Internet]. $2018 \mathrm{Apr}$ 1 [cited 2019 Sep 7];3(4):346. Available from: http://cardiology.jamanetwork.com/article. aspx?doi=10.1001/jamacardio.2018.0001

2. Torino C, Gargani L, Sicari R, Letachowicz K, Ekart R, Fliser D, et al. The agreement between auscultation and lung ultrasound in hemodialysis patients: The LUST Study. Clin J Am Soc Nephrol [Internet]. 2016 Nov 7 [cited 2019 Sep 7];11(11):2005-11. Available from: http://cjasn.asnjournals. org/lookup/doi/10.2215/CJN.03890416

3. Picano E, Pellikka PA. Ultrasound of extravascular lung water: A new standard for pulmonary congestion. Eur Heart J [Internet]. 2016 Jul 14 [cited 2019 Sep 7];37(27):2097-104. Available from: https://academic.oup.com/eurheartj/article-lookup/doi/10.1093/eurheartj/ehw164

4. Stawicki SP, Braslow BM, Panebianco NL, Kirkpatrick JN, Gracias VH, Hayden GE, et al. Intensivist use of hand-carried ultrasonography to measure IVC collapsibility in estimating intravascular volume status: Correlations with CVP. J Am Coll Surg [Internet]. 2009 Jul [cited 2019 Sep 7];209(1):55-61. Available from: https://linkinghub.elsevier.com/retrieve/pii/S1072751509002865

5. Mullangi S, Sozio SM, Hellmann DB, Martire C, Lohani S, Segal P, et al. Integrative point-of-care ultrasound curriculum to impart diagnostic skills relevant to nephrology. Am J Kidney Dis [Internet]. 2019 Jun [cited 2019 Sep 7];73(6):894-6. Available from: https://linkinghub.elsevier.com/retrieve/ pii/S027263861930126X

6. Koratala A, Segal MS, Kazory A. Integrating point-of-care ultrasonography into nephrology fellowship training: A model curriculum. Am J Kidney Dis [Internet]. 2019 Jul [cited 2019 Sep 7];74(1):15. Available from: https://linkinghub.elsevier.com/retrieve/pii/S0272638619301295

7. Rivas-Lasarte M, Maestro-Benedicto A, Fernandez-Martinez J, Álvarez-García J, Solé-González E, López-López L, et al. Lung ultrasound may reduce heart failure hospitalizations: Preliminary results from the LUS-HF Trial. J Heart Lung Transplant [Internet]. 2019 Apr [cited 2019 Sep 8];38(4):S141. Available from: https://linkinghub.elsevier.com/retrieve/pii/S1053249819303377

8. Loutradis C, Sarafidis PA, Ekart R, Papadopoulos C, Sachpekidis V, Alexandrou ME, et al. The effect of dry-weight reduction guided by lung ultrasound on ambulatory blood pressure in hemodialysis patients: A randomized controlled trial. Kidney Int [Internet]. 2019 Jun [cited 2019 Sep 8];95(6):1505-13. Available from: https://linkinghub.elsevier.com/retrieve/pii/S0085253819302704

\section{Correspondence to:}

Hugo Fernandes Diniz, MD

Serviço de Nefrologia, Centro Hospitalar e Universitário de São João, Alameda Prof. Hernâni Monteiro, 4200-319 Porto

E-mail: hugo.rc.dz@gmail.com 